\title{
Universal Real-time Monitoring System Based on Ethernet in Test and Measurement Domain
}

\author{
Ma Zhigang ${ }^{1,2}$, Liu Wenyi ${ }^{1, *}$ and Zhang Wendong ${ }^{1}$
}

${ }^{1}$ Key Laboratory of Instrumentation Science \& Dynamic Measurement (North University of China), Ministry of Educa-
tion; Science and Technology on Electronic Test \& Measurement Laboratory, Taiyuan, Shanxi, 030051, P.R. China
${ }^{2}$ College of Information Science and Engineering, Shanxi Agricultural University, Taigu, Shanxi, 030801, P.R. China

\begin{abstract}
In test and measurement systems, real-time monitoring is the effective method for obtaining the running conditions and making fault diagnosis in testing system. In this paper, a universal real-time monitoring system based on User Datagram Protocol (UDP) is discussed. Firstly, the component structure of monitoring system is introduced, and comparisons of various kinds of data transmission modes between the front-end acquisition module and the back-end monitoring module are made. Secondly, the characteristics and data communication mode of UDP are discussed, and the general process of real-time data analysis and curve plotting are studied. Meanwhile, a multi-channel data acquisition and monitoring system application instance for aerospace testing is designed and analyzed. It is demonstrated that the proposed design method can satisfy the test requirements, and because of the universality, which can be applied to other test fields by means of appropriate transformation or revise.
\end{abstract}

Keywords: Data transmission, Ethernet, Real-time monitoring, Test and measurement, User Datagram Protocol (UDP).

\section{INTRODUCTION}

Presently, with the rapid development of computer hardware and software, sensors, communications and other related technologies, real-time monitoring technologies and systems have been widely applied to several domains, such as: monitoring and fault diagnosis of industrial Equipments [2], earthquake [3], geological disaster prediction [4], construction health monitoring [5], power systems monitoring and maintenance $[6,7]$ (supply, distribution, transformation of the power, etc.), energy resources [8-[10]; food safety, environmental monitoring (water quality, air-quality [11], illumination [12], noise [13], pernicious gas, respirable content particles), weather forecasting; medical monitoring [14], transportation, security maintenance; aviation and aerospace [15], and so on. All types of real-time monitoring systems play important and indispensable role in ensuring the rapid development of industrial and agricultural production, maintenance of environmental safety, aerospace, and national defense.

Real-time monitoring system is usually composed of the front-end acquisition module and the back-end (remote) monitoring module; there are several data communication ways between the front-end and the back-end. For example: the literature [15] designed a rocket parameters real-time monitoring system using USB bus; in the literature [4] and [8], optical fiber was applied to monitoring the hill landslide and the mine temperature respectively; the literature [16] studied the synchronization and real-time transmission problems in Ethernet distributed data acquisition; the literature [5] and [7] applied Ethernet distributed transmission to the dam and power system monitoring respectively; the literature [14] employed public wireless communication network to design the remote medical monitoring and analysis terminal device.

Based on the analysis of the characteristics of various communication ways, the paper discusses the general method of constructing real-time monitoring system based on Ethernet data transmission, and the validity and reliability of the method is verified by an application instance.

\section{REAL-TIME MONITORING SYSTEM}

In real-time monitoring system, a large number of testing data acquired from the front-end module will be transmitted to back-end monitoring module. The data can reflect the running status and performance parameters of the equipments or systems; the analysis, computation and storage of which, can provide the decision-making basis for the equipments or system. A real-time monitoring system is composed of front-end acquisition module and back-end monitoring module, in order to establish the communication link between the two modules, wireless or transmission cable should be adopted, which is shown in Fig. (1).

No matter which communication way is applied between the front-end module and the back-end module, it accords to their respective characteristics and applicability. By contrast: USB can transfer faster, such as USB2.0 and USB3.0 can 


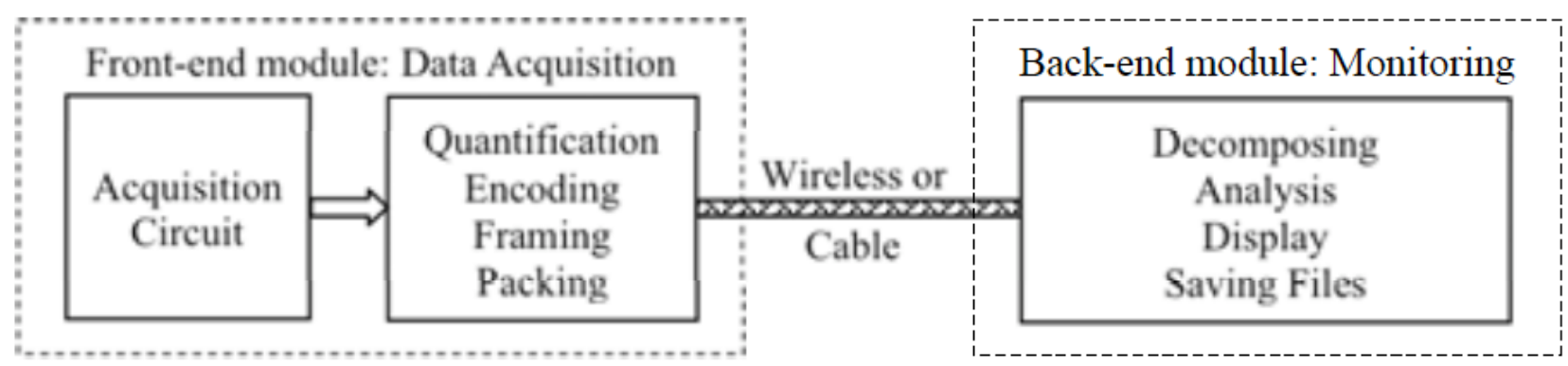

Fig. (1). Real-time monitoring system.

\begin{tabular}{|l|l|l|l|l|}
\hline Head & Data Content & Counter & Checksum Word & Tail \\
\hline
\end{tabular}

Fig. (2). Frame or packet structure.

transmit data up to $480 \mathrm{Mbps}$ and $5 \mathrm{G}$ bps respectively, but the transmission distance is limited and susceptible to electromagnetic environment interference, which is possible to lead to disconnection. The optical fiber is not susceptible to complex electromagnetic environment, and data transmission is faster, that is the data transmission of rate single optical fiber can be up to several Gbps. Meanwhile, the transmission distance can be up to several tens of kilometers. However the fiber is more fragile and easily broken, so it needs to be lay buried, which is inconvenient. For Ethernet data transmission way, the hardware and software structure are simpler, and the transmission distance can up to 100 meters, but there is a risk of data loss in UDP transmission mode. Wireless sensor networks (WSN) can be used for multi-node monitoring system, while the multi-node time synchronization, coordination and other troublesome problems should be deliberated. The existing public wireless communication network can be applied directly to data communication, and its hardware cost is lower, but it is necessary to pay attention to the strength and coverage of the wireless signal.

Based on the above comparisons, if there are several back-end modules for monitoring different kinds of parameters, the industrial Ethernet data transmission format may be more suitable. At the same time, because of the simple hardware and software design process, Ethernet transmission mode is widely used in several kinds of industrial control and aerospace testing fields in recent years.

\section{REAL-TIME MONITORING SYSTEM BASED ON ETHERNET}

Transmission Control Protocol (TCP) and User Datagram Protocol (UDP) are two basic methods of Ethernet communication. TCP is a reliable transmission protocol, which is designed for Wide Area Network (WAN) and based on connection. Before the data transmission begins, a connection between the transmitter and receiver is must be established firstly, and all the transmitted message is should be confirmed by the receiver. UDP is a simple connectionless protocol, which provides connectionless and unreliable data transmission service. However, in some exclusive local area network (LAN), UDP has following special advantages [17]:
(1) It is unnecessary to maintain connection status, the data receipt confirmation or time-out retransmission mechanisms when the data is transmitting, therefore the transmission speed is much faster than TCP. Furthermore, UDP also supports broadcast and multicast data transmission.

(2) The data packet header of UDP is only 8 bytes (B), much less than 20 bytes of TCP, so UDP has lower consumption rate. At the same time, because of packet-oriented, there is no data fragmentation or connection management, which will reduce the system expense and has more effective utilization ratio of communication bandwidth than TCP.

(3) Data throughput capacity is not controlled by the congestion control algorithm, only affected by the data generated speed of application program, the performance of computers and the transmission bandwidth.

Therefore, for some exclusive LANs which have good quality network environment and reliability, UDP is more appropriate.

\subsection{Data Framing and Package}

The test data, obtained by acquisition circuit, must be framed and packed firstly, and then can be sent to the backend monitoring module. Different testing data has its own specific organization pattern, which was entitled "framing", and a framed data was called as "frame structure". "Package" is a combination of several data frames, and the packet will be transmitted to the back-end module. The packet size depends on the sampling rate, data size, processing time and other factors. "Frame structure" or "package structure" is usually composed of frame (or packet) header, data content, frame (or packet) counter, checksum word, frame (or packet) tail and other parts. Frame or packet structure is shown in Fig. (2).

For example, the data acquired in every millisecond (ms, $0.001 \mathrm{~s}$ ) can be constructed to one frame, and then the data in $100 \mathrm{~ms}(0.1 \mathrm{~s})$ would be packed as one packet (100 frames), after that, the packet can be sent to the back-end monitoring module. Thus, the monitoring module will receive and process a packet data per $0.1 \mathrm{~s}$. 


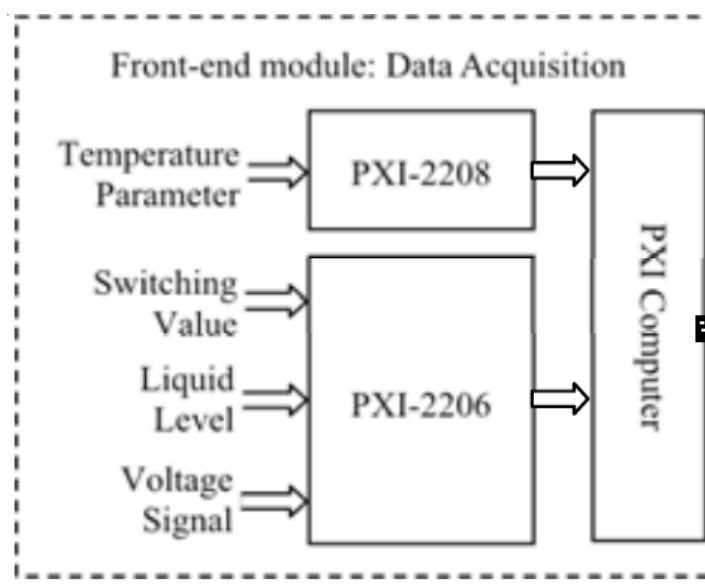

Fig. (3). The multi-channel real-time monitoring system based on UDP.

\subsection{Data Packet Sending and Receiving}

By means of UDP, data can be sent to (or received from) the local computer specified by its internet protocol (IP) address. Before the data communication, both the IP addresses and port numbers of local and remote computer are must be specified in advance. During the data communication, the data is identified by computer's IP address and port number. A packet of data affixed with receiver's IP address and port number will be sent form the sender to the receiver, meanwhile, the receiver can receive the data in buffer area by the timing or polling method [7][17].

In Visual $\mathrm{C}++$, UDP data transmission program can be implemented easily by calling functions encapsulated in the Class named as "winsock", that is: socket(), bind(), sendto(), recvfrom(), closesocket(), WSACleanup(), and so on. The data sending process is:

(1) Constructing a socket by calling socket() function;

(2) Binding the socket to the local computer which is specified by its IP address and port number by Calling bind() function;

(3) Sending timing data packets to the receiver by calling sendto() function.

When receiving the data packets, a socket is should be constructed and bind firstly. After that, the data packets can be received by Calling recvfrom() function. When the data communication is finished, the socket is must be closed and the resource is should be released by Calling closesocket() function. Finally, using of the socket will be terminated by Calling WSACleanup () function.

\subsection{Data Processing and Curve Plotting}

The data received by the back-end monitoring module needs to be "unpacked" and "de-framed" firstly, and then every channel data can be obtained, after necessary processing and computation, the channel data will be displayed in the form of text or curve on the monitoring interface. Since the data curve is the most intuitive method for processing and describing the monitoring data, the dynamic real-time data curve is commonly used in several testing system, which can show the change of critical parameters conveniently; at the same time, the curve also reflects the state of the monitored object or system, which will play a significant role in the fault diagnosis.

In order to fulfill the real-time processing requirement, besides of storage structure of the data, the control structure of the program, the amount of processed data, etc, the programming development tool is also should be deliberated. In current common programming languages, $\mathrm{C} / \mathrm{C}++$ language is generally considered as the relatively higher efficient one, combined with related function in API can largely improve the efficiency of data processing and curve plotting. In order to achieve a complete analysis of the data, if there is some surplus data in tail of the prior data packet, it should be cached firstly, and then joined with the next packet data before analysis.

\section{APPLICATION INSTANCE AND ITS ANALYSIS}

According to the demand of an aerospace testing project, a multi-channel data acquisition and real-time monitoring system is required to design. There are 74 parameters need be acquired and monitored, that is: 60 temperature parameter, 5 switch value, 5 liquid level and 4 voltage signal. Among of which, temperature, switch signal and liquid level, voltage signal would be analyzed, displayed in two individual back-end modules; and the distance between the front-end module and back-end ones is about 70 meters (m).

\subsection{System Design Scheme}

In order to simplify the design procedure, a PXI computer is applied to acquire and transmit data; PXI-2008 and PXI2006 are chosen for acquisition cards. Among of the two cards, the PXI-2008 card is responsible for 60 temperature signals' acquisition, while the PXI-2006 card takes charge to acquire 5 switching value, 5 liquid level and 4 voltage signals. Between the front-end acquisition module and two back-end monitoring modules, there is a switching equipment to transmit data by the Gigabit Ethernet cable. When the data is received by the back-end module, data unpacking, analysis, display, channel data export and saving files will be in progress. The system component of multi-channel real-time monitoring system based on UDP is shown in Fig. (3). 


\begin{tabular}{|c|c|c|c|c|c|c|c|c|c|}
\hline \multirow{2}{*}{$\begin{array}{c}\text { Frame Head } \\
\text { (2Bytes) } \\
\text { 0X"DC92" }\end{array}$} & \multicolumn{6}{|c|}{ Data (125 Bytes ) } & \multirow{2}{*}{$\begin{array}{c}\text { Frame } \\
\text { Counter } \\
\text { (4 Bytes) }\end{array}$} & \multirow{2}{*}{$\begin{array}{l}\text { CRC Sum } \\
\text { Word } \\
\text { (2 Bytes) }\end{array}$} & \multirow{2}{*}{$\begin{array}{c}\text { Frame Tail } \\
\text { (2Bytes) } \\
\text { 0X"EB90" }\end{array}$} \\
\hline & $\begin{array}{c}\text { Temperature } 1 \\
\text { (2 Bytes) }\end{array}$ & $\cdots$ & $\begin{array}{c}\text { Temperature } 60 \\
\text { (2 Bytes) }\end{array}$ & $\begin{array}{c}\text { switching } \\
\text { value } 1 \\
\text { (1 Bytes) }\end{array}$ & $\cdots$ & $\begin{array}{l}\text { switching } \\
\text { value } 5 \\
\text { (1 Bytes) }\end{array}$ & & & \\
\hline
\end{tabular}

Fig. (4). The frame structure of temperature and switching value.

\begin{tabular}{|c|c|c|c|c|c|c|c|c|c|}
\hline \multirow{2}{*}{$\begin{array}{c}\text { Frame Head } \\
\text { (2Bytes) } \\
0 X^{\prime \prime} 146 F^{\prime \prime}\end{array}$} & \multicolumn{6}{|c|}{ Data (18 Bytes ) } & \multirow{2}{*}{$\begin{array}{c}\text { Frame } \\
\text { Counter } \\
\text { (4 Bytes) }\end{array}$} & \multirow{2}{*}{$\begin{array}{c}\text { CRC Sum } \\
\text { Word } \\
\text { (2 Bytes) }\end{array}$} & \multirow{2}{*}{$\begin{array}{c}\text { Frame Tail } \\
\text { (2Bytes) } \\
\text { 0X"09BE" }\end{array}$} \\
\hline & $\begin{array}{l}\text { Liquid Level } 1 \\
\quad \text { (2 Bytes) }\end{array}$ & $\cdots$ & $\begin{array}{l}\text { Liquid Level } 5 \\
\quad(2 \text { Bytes })\end{array}$ & $\begin{array}{l}\text { Voltage } 1 \\
\text { (2 Bytes) }\end{array}$ & $\cdots$ & $\begin{array}{l}\text { Voltage } 4 \\
\text { (2 Bytes) }\end{array}$ & & & \\
\hline
\end{tabular}

Fig. (5). The frame structure of liquid level and voltage data.

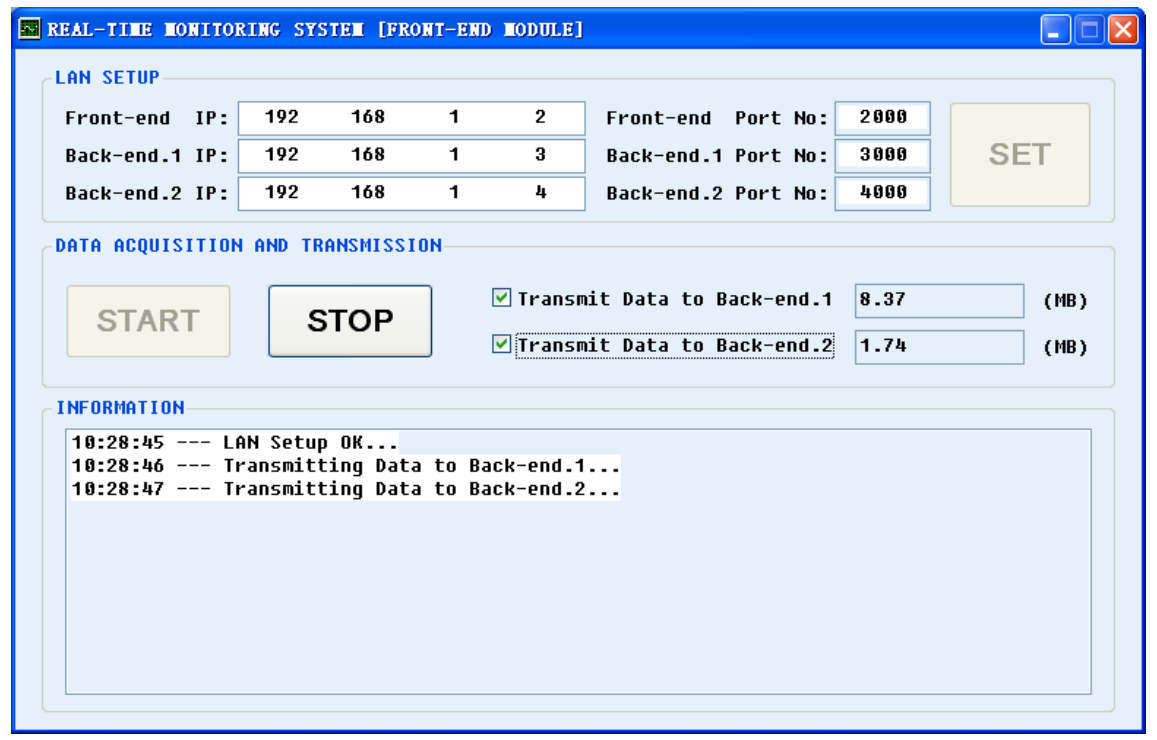

Fig. (6). The interface of front-end acquisition module.

\subsection{Framing and Package}

Since there are two back-end monitoring modules in the system, the front-end module is required to generate two kinds of data packet and sent them to each back-end module respectively. The frame structure including of temperature and switching data is shown in Fig. (4). In similar, the frame structure including of liquid level and voltage data is shown in Fig. (5). In order to ensure the transmission reliability, the CRC sum word is set in two frame structures. If the back-end monitoring module calculates $\mathrm{CRC}$ checksum incorrect, it will discard the frame data. Generally speaking, minute quantity error frame data will have little impact on the overall system, so it could be neglected in some permitted conditions.

According to the design request, all the monitored parameters are slow variables. Each acquisition channel is set as $1 \mathrm{kHz}$ sampling rate and 16 bits (b) sampling digit. Meanwhile, data packet will be transmitted per $100 \mathrm{~ms}$. Therefore, the packet size of temperature and switching data is 13500 bytes, while the packet size of liquid level and voltage data is 2800 bytes.

\subsection{Programming of Acquisition and Transmission}

In the front-end module, data acquisition is mainly accomplished by a PXI computer and two cards (PXI-2008 and
PXI-2006), besides of necessary peripheral circuits (power supply, filtering, conditioning circuits, etc.), the major work needs to be done by programming in the PXI computer.

Because of using two acquisition cards, it is indispensable to paid more attention to the synchronous acquisition problem. Since the two cards have the same data sampling rate $(1 \mathrm{kHz})$, if they can be start to acquire simultaneously, they will complete an acquisition operation at the same time. In programming, the two cards are configured as double buffering mode. Due to the same sampling rate, the two cards' data can be obtained simultaneously in the light of the half-full message coming from either card. In order to ensure sending packets on time, "Acquisition" $\rightarrow$ "Package" $\rightarrow$ "Sending" should be implemented in cycle mode.

\subsection{Software Design and Test Results}

Based on the functional requirements of the real-time system, a small LAN real-time monitoring system software for the aerospace testing is developed based on $\mathrm{VC}++2008$ (VC 9.0). The software interface of front-end acquisition module and back-end monitoring module 1(temperature and switching value) are shown in Fig. (6) and Fig. (7) respectively. Because of the similarity between the back-end monitoring module 1 and 2, interface of module 2 is leave out here. 


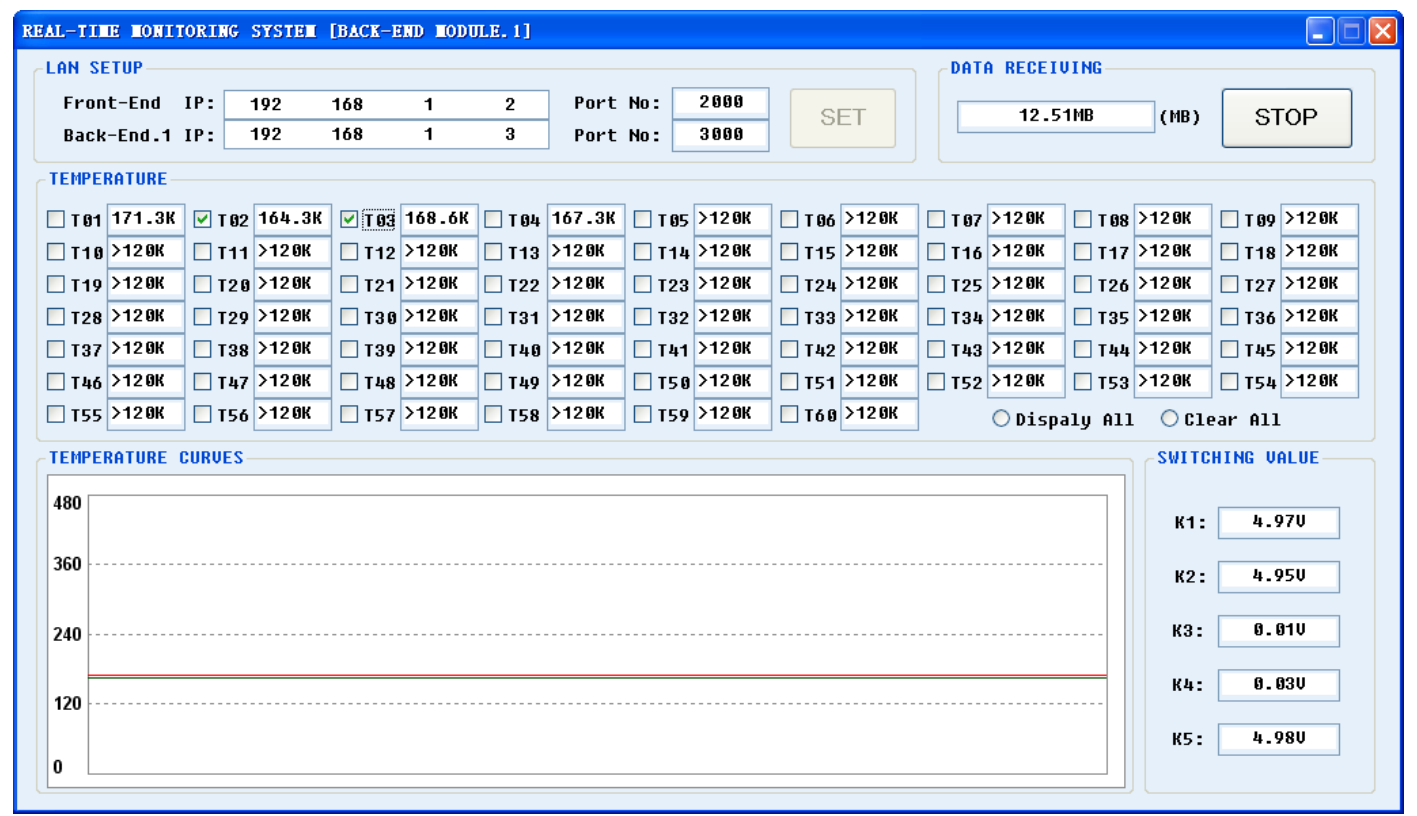

Fig. (7). The interface of back-end monitoring module 1.

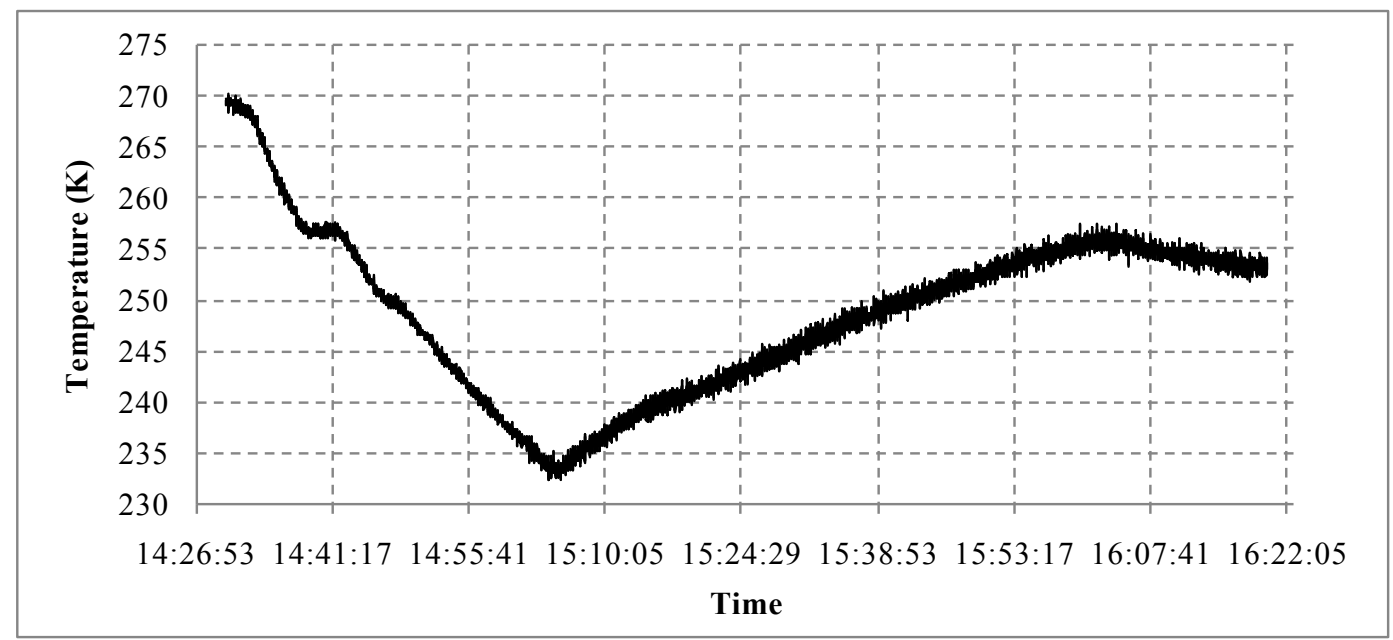

Fig. (8). Temperature playback curve of a testing point.

In Fig. (8), there are two temperature curves corresponding to channel-2 (T02) and channel-3 (T03) respectively. Since the temperature is a kind of slowly changing signal, the curve shows nearly linear state in a short time. Fig. 10 is a temperature playback curve of a testing point (displaying one data point per second), the unit is Kelvin (K), which last about 1.5 hours.

\section{CONCLUSION}

Various real-time monitoring tools have provided great guarantee of reliability and security for testing system. The general design process of the Ethernet data transmission and real-time monitoring system is studied in this paper. Based on the analysis of various types of data communication, UDP method is considered having following advantages: simple structure of monitoring system, relatively easily programming, lower cost, and so on. Therefore, UDP is suitable for small LAN monitoring system. Owing to the distributed feature of Ethernet, the multi-node monitoring system can be constructed, in which every node is relatively independent (the monitoring data is different) and belongs to the same LAN. The real-time monitoring system is widely applied in various testing areas. The construction method of the monitoring system proposed in this paper has obtained several successful applications in a number of aerospace testing projects and achieved satisfactory testing results. Although the requirements of different testing domains in real-time monitoring are distinct, the method discussed has some universality, and can be applied to the general applications with appropriate improvement or revise.

\section{CONFLICT OF INTEREST}

The authors declare that there is no conflict of interest regarding the publication of this article. 


\section{ACKNOWLEDGEMENTS}

This work is supported by the National Natural Science Foundation of China (5127549).

\section{REFERENCES}

[1] X. J. Zhou, W. B. Shen, and L. F. Xi, "An adaptive sampling algorithm for real time monitoring of equipments", Journal of Shanghai Jiaotong University, vol. 42, pp. 1975-1978, Dec. 2008.

[2] A. Uysal, and R. Bayir, "Real-time condition monitoring and fault diagnosis in switched reluctance motors with Kohonen neural network", Journal of Zhejiang University-SCIENCE C (Computers \& Electronics), vol. 14, pp. 941-952, Dec. 2013.

[3] X. X. Hu, Y. T. Teng, and X. Z. Wang, X. Wang, Y. Zhang, and C. Wang, "The design of real-monitor software earthquake acquisition system", Seismological and Geomagnetic Observation and Research, vol. 29, pp. 104-108, Jan. 2008.

[4] Y. J. Sun, D. Zhang, H. J. Tong, and B. Shi, "Research of distributed fiber optic sensing technology in monitoring of Majiagou landside of Three Gorges", The Chinese Journal of Geological Hazard and Control, vol. 24, pp. 97-102, Apr. 2013.

[5] B. Y. Guo, Z. Li, S. and Q. Hua, "Earthquake message triggered dam real-time monitoring system based on reliable UDP communication", Hydropower Automation and Dam Monitoring, vol. 35, pp. 57-60, May. 2011.

[6] H. Wei, L. Wang, T. Zhao, Y. Liu, and F. Li, "Intelligent terminal of substation equipment condition monitoring based on UDP", Science Technology and Engineering, vol. 12, pp. 5888-5902, Aug. 2012.

[7] S. B. Wu, and W. Zhu, "Design of surge protection monitoring system based on the UDP network protocol", Journal of Shanghai University of Electric Power, vol. 29, pp. 283-287, Mar. 2013.
[8] C. Lu, "Design of distributed located mine temperature monitoring and measurement system", Coal Science and Technology, vol. 35, pp. 51-54, Dec. 2007.

[9] W. Yang, and D. Z. Zhang, "Multi-parameters monitoring underground coal mine environment using mesh-structured wireless sensor networks", Journal of Huazhong University of Science Technology (Natural Science Edition), vol. 38, pp. 70-74, Oct. 2010.

[10] X. M. Liu, J. LV, W. Q. Chu, R. Hu, and A. Liu, "The downhole fracture real-time monitoring technology and its application", China Petroleum Machinery, vol. 40, pp. 101-104, May. 2012.

[11] Y. Q. Li, B. Y. Yang, and L. P. Zhang, "Air quality monitoring system based on industrial Ethernet", Machine Tool \& Hydraulics, vol. 37, pp. 152-155, Jun. 2009.

[12] G. J. Wang, J. M. Bao, S. Li, et al. "Research and realization of distributed monitoring system environment illumination", Process Automation Instrumentation, vol. 34, pp. 34-36, Sep. 2013.

[13] X. Leng, J. F. Chen, and Y. Yong, "A distributed environment sound and vibration monitoring system", Measurement \& Control Technology, vol. 32, pp. 24-27, Jun. 2013.

[14] N. Zhang, and Y. Zhang, "Development for a remote medical realtime monitoring and analysis fronted device", Automation \& Instrumentation, vol. 25, pp. 5-8, Feb. 2010.

[15] S. M. Shen, S. Z. Di, Q. Li, J. Xiong, and W. Zhang, "Design of real-time monitoring system base on FPGA for rocket parameters", Fire control \& Command Control, vol. 36, pp. 160-163, May. 2011.

[16] X. F. You, Y. M. Wen, and P. Li, "Study on the synchronization of distributed data acquisition and real-time transmission based on Ethernet", Chinese Journal of Scientific Instrument, vol. 27, pp. 384-388, Apr. 2006.

[17] H. Y. Zhuo, J. Chen, and J. R. Zhang, "Realization of distributed and real-time monitor system based on UDP protocol under VC environment", Ordnance Industry Automation, vol. 27, pp. 85-86, Dec. 2008.

Received: September 22, 2014

Revised: November 03, 2014

Accepted: November 06, 2014

(C) Zhigang et al.; Licensee Bentham Open.

This is an open access article licensed under the terms of the Creative Commons Attribution Non-Commercial License (http://creativecommons.org/licenses/by-nc/3.0/) which permits unrestricted, non-commercial use, distribution and reproduction in any medium, provided the work is properly cited. 